\title{
Gyroid Nanoporous Membranes with Tunable Permeability
}

\author{
Li, Li; Schulte, Lars; Clausen, Lydia D.; Hansen, Kristian M.; Jonsson, Gunnar Eigil; Ndoni, Sokol
}

Published in:

A C S Nano

Link to article, DOI:

$10.1021 / \mathrm{nn} 200610 \mathrm{r}$

Publication date:

2011

Document Version

Publisher's PDF, also known as Version of record

Link back to DTU Orbit

Citation (APA):

Li, L., Schulte, L., Clausen, L. D., Hansen, K. M., Jonsson, G. E., \& Ndoni, S. (2011). Gyroid Nanoporous Membranes with Tunable Permeability. A C S Nano, 5(10), 7754-7766. https://doi.org/10.1021/nn200610r

\section{General rights}

Copyright and moral rights for the publications made accessible in the public portal are retained by the authors and/or other copyright owners and it is a condition of accessing publications that users recognise and abide by the legal requirements associated with these rights.

- Users may download and print one copy of any publication from the public portal for the purpose of private study or research.

- You may not further distribute the material or use it for any profit-making activity or commercial gain

- You may freely distribute the URL identifying the publication in the public portal

If you believe that this document breaches copyright please contact us providing details, and we will remove access to the work immediately and investigate your claim 


\title{
Gyroid Nanoporous Membranes with Tunable Permeability
}

\author{
Li Li, ${ }^{\dagger}$ Lars Schulte, ${ }^{\ddagger}$ Lydia D. Clausen, ${ }^{\S}$ Kristian M. Hansen, ${ }^{\S}$ Gunnar E. Jonsson," and Sokol Ndoni ${ }^{\ddagger * *}$ \\ ${ }^{\dagger}$ Danish Polymer Centre, Department of Chemical and Biochemical Engineering, ${ }^{\ddagger}$ Department of Micro and Nanotechnology, "Computer Aided Process \\ Engineering Center, Department of Chemical and Biochemical Engineering, Technical University of Denmark, DK-2800 Kgs. Lyngby, Denmark, and ${ }^{\S}$ Radiometer \\ Medical ApS, DK-2700 Brønshøj, Denmark
}

M embranes from nanoporous materials in general and from nanoporous polymers in particular are of great interest for a variety of applications in the health sector, food industry, sustainable water treatment, and energy conversion. ${ }^{1-4}$ Size-sieving and transport of molecules in a membrane is a crucial step for these applications. Advances in nanofabrication make it possible to accurately control morphology, pore size, and surface chemistry of nanoporous materials, allowing better predictability of mass transport at the molecular level.

Two of the most common techniques that are used to create nanoporous polymeric films are phase separation ${ }^{5}$ and ion-track etching. ${ }^{6}$ Commercial membranes fabricated by phase separation generally exhibit broad pore size distribution and relatively high thickness. As a result, these materials possess poor size-cutoff properties and low transport rates. lon-track etched membranes show a better size cutoff, but their porosity is too low for most practical uses. Alternatively, anodized nanoporous materials, ${ }^{7}$ for example, nanoporous alumina and nanoporous silica, have gained considerable attention due to the well-ordered cylindrical pores and narrow pore size distribution. However, weak mechanical properties limit their resistance to high pressure in a convection mode. It is also difficult to manufacture a device out of these brittle materials.

Block copolymer-templated nanoporous materials are being extensively developed. Due to the incompatibility of the constituent blocks, the block copolymers self-assemble into arrays of various well-defined structures, such as spheres, cylinders, lamellae, or more complex morphologies, with a microdomain dimension in the molecular length scale. ${ }^{8}$ Nanoporous matrices can be derived from self-assembled block copolymers by partially or totally removing one
ABSTRACT Understanding the relevant permeability properties of ultrafiltration membranes is facilitated by using materials and procedures that allow a high degree of control on morphology and chemical composition. Here we present the first study on diffusion permeability through gyroid nanoporous cross-linked 1,2-polybutadiene (1,2-PB) membranes with uniform pores that, if needed, can be rendered hydrophilic. The gyroid porosity has the advantage of isotropic percolation with no need for structure prealignment. Closed (skin) or opened (nonskin) outer surface can be simply realized by altering the interface energy in the process of membrane fabrication. The morphology of the membranes' outer surface was investigated by scanning electron microscopy, contact angle, and $X$-ray photoelectron spectroscopy. The effective diffusion coefficient of glucose decreases from nonskin, to one-sided skin to two-sided skin membranes, much faster than expected by a naive resistance-in-series model; the flux through the two-sided skin membranes even increases with the membrane thickness. We propose a model that captures the physics behind the observed phenomena, as confirmed by flow visualization experiments. The chemistry of 1,2-PB nanoporous membranes can be controlled, for example, by hydrophilic patterning of the originally hydrophobic membranes, which allows for different active porosity toward aqueous solutions and, therefore, different permeability. The membrane selectivity is evaluated by comparing the effective diffusion coefficients of a series of antibiotics, proteins, and other biomolecules; solute permeation is discussed in terms of hindered diffusion. The combination of uniform bulk morphology, isotropically percolating porosity, controlled surface chemistry, and tunable permeability is distinctive for the presented gyroid nanoporous membranes.

KEYWORDS: block copolymer · surface morphology · skin layer $\cdot \mathrm{HPL} \cdot$ defects $\cdot$ diffusion · selectivity

block with UV, ${ }^{9}$ oxygen plasma, ${ }^{10}$ ozone, ${ }^{11}$ base, ${ }^{12}$ acid $^{13,14}$ or fluorine compounds. ${ }^{14-17}$ Alternatively, block copolymers and nanoporous matrices derived from them have been used to direct the morphology of, for example, silicas and metal oxides. ${ }^{18}$ We successfully generated a series of nanoporous polymers by selectively and quantitatively etching polydimethylsiloxane (PDMS) with anhydrous hydrogen fluoride, ${ }^{14-16}$ trifluoroacetic acid, ${ }^{14}$ or tetrabutyl ammonium fluoride. ${ }^{16,17}$ Unique features like controllable morphology, pore size and orientation, high porosity, narrow pore size distribution, ${ }^{17}$ and easy surface functionalization ${ }^{19-21}$ render, especially the nanoporous polydiene materials, very attractive for many membrane applications. ${ }^{22}$ *Address correspondence to
sokol.ndoni@nanotech.dtu.dk.

Received for review February 14, 2011 and accepted August 25, 2011.

Published online August 25, 2011 10.1021/nn200610r

C 2011 American Chemical Society 


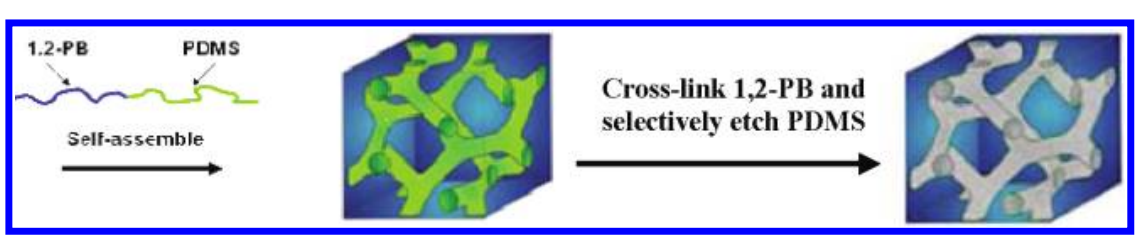

Figure 1. Schematic illustration of the fabrication steps of cross-linked nanoporous 1,2-PB polymer from a 1,2-PB-b-PDMS block copolymer precursor. The precursor (left) self-assembles into a gyroid morphology at the cross-linking temperature (middle). PDMS is selectively cleaved from the sample and a nanoporous polymer matrix is obtained (right).

Nanoporous polymer membranes derived from block copolymers show high potential as efficient separation membranes. ${ }^{23}$ Russell et al. ${ }^{24}$ fabricated an asymmetric membrane for the filtration of viruses where a thin nanoporous layer from polystyrene- $b$ poly(methyl methacrylate) (PS- $b$-PMMA) functioned as a top-layer membrane with ultrahigh selectivity and flux. Seung et al. ${ }^{25}$ developed a new protein drug delivery device using membranes with cylindrical nanopores derived from PS- $b$-PMMA. The pore size of the PS nanochannels could be tuned from 15 to $6 \mathrm{~nm}$ by gold deposition. A nanoporous PS thin film fabricated from polystyrene- $b$-poly(L-lactide) was used as a template to load sirolimus; it was then applied as a coating on implantable devices for drug delivery. ${ }^{26}$ Hillmyer et al. ${ }^{27}$ reported ultrafiltration studies of a series of nanoporous membranes based on block copolymers for water treatment. In addition, a combination of molecular size selectivity in the nanoscale and superior mechanical properties in the macroscale were demonstrated for nanoporous polyethylene membranes prepared from polyethylene- $b$-polystyrene precursor. $^{28}$

More interestingly, while the bulk morphology of block copolymers can be controlled by composition, molecular architecture, chain length, and temperature, the outer surface morphology can be manipulated by controlling interfacial energy. ${ }^{8,29}$ This can in turn affect the properties and performance of the resultant nanoporous membrane, such as permeation rate, selectivity and biocompatibility. We fabricated nanoporous membranes from self-assembled 1,2-polybutadiene- $b$-polydimethylsiloxane (1,2-PB- $b$-PDMS) via quantitative and selective cleavage of the PDMS block, as illustrated in Figure 1. A gyroid nanostructure was designed from the polymer synthesis stage ${ }^{17}$ to ensure isotropic percolation with no need for structure prealignment. The membrane has narrow pore size distribution and high porosity, so high throughput and selectivity are expected. We selected two different substrates to interface-direct two distinct morphologies at the outer surface of the nanoporous membranes. The surface morphology was comprehensively investigated by scanning electron microscopy (SEM), atomic force microscopy (AFM), contact angle (CA), and X-ray photoelectron spectroscopy (XPS). We demonstrate the effect of surface morphology and membrane thickness on permeation rate by testing glucose diffusion through the membranes. The originally hydrophobic membrane can be hydrophilized via UV photo-oxidation ${ }^{20}$ or thiol-ene photochemistry. ${ }^{21}$ We selectively hydrophilized controlled portions of the membrane by utilizing patterned UV masks, thus, rendering tunable active porosity toward aqueous solutions. The selectivity of the membranes was further assessed by measuring the diffusive transport of a series of antibiotics, proteins and other biomolecules.

\section{RESULTS AND DISCUSSION}

The section is composed of two main parts, the first focusing on the structural characterization of the membranes, and the second on their performance in diffusive transport regime. We start the characterization part by demonstrating the gyroid bulk morphology, touching also on the subject of pore connectivity across grain boundaries in the bulk. A detailed study of the surface morphology follows, evidencing the role of substrate on the absence or presence of skin layer, its structural relation to the microphases of the block copolymer precursor, and its possible defects. First in the performance part we present and conceptually model the diffusion of glucose through gyroid membranes with no skin layer and with skin layer on one or both sides. Then, it is demonstrated that the active porosity for permeation of water solutions can be controlled by patterned hydrophilization of the membranes. At the end, the selectivity of the nonskin membranes in diffusive flow is studied relative to a number of biologically relevant molecules and proteins; the results are analyzed in terms of hindered diffusion.

\section{Membrane Characterization}

Bulk Morphology. Figure 2a shows a typical nanoporous 1,2-PB membrane prepared in this study. It is colorless, transparent, and flexible. The bulk morphology of the membranes was imaged by SEM and TEM, as shown in Figures $2 b, c$ and 3. It was further confirmed by small-angle X-ray scattering (SAXS) and AFM, as can be seen in Figures $\mathrm{S} 1$ and $\mathrm{S} 2 \mathrm{a}$ of the Supporting Information (SI). Both the SEM and TEM images show regular patterns with uniform pore size of $\sim 10 \mathrm{~nm}$ in diameter and a crystallographic unit cell size of $45 \mathrm{~nm}$ (see also Figure S1). The crystal-like structures of Figure 2 are characteristic within each gyroid grain; the membrane as a whole is a "polycrystalline" material with grain sizes in the range of $1-5 \mu \mathrm{m}$, as can be seen 


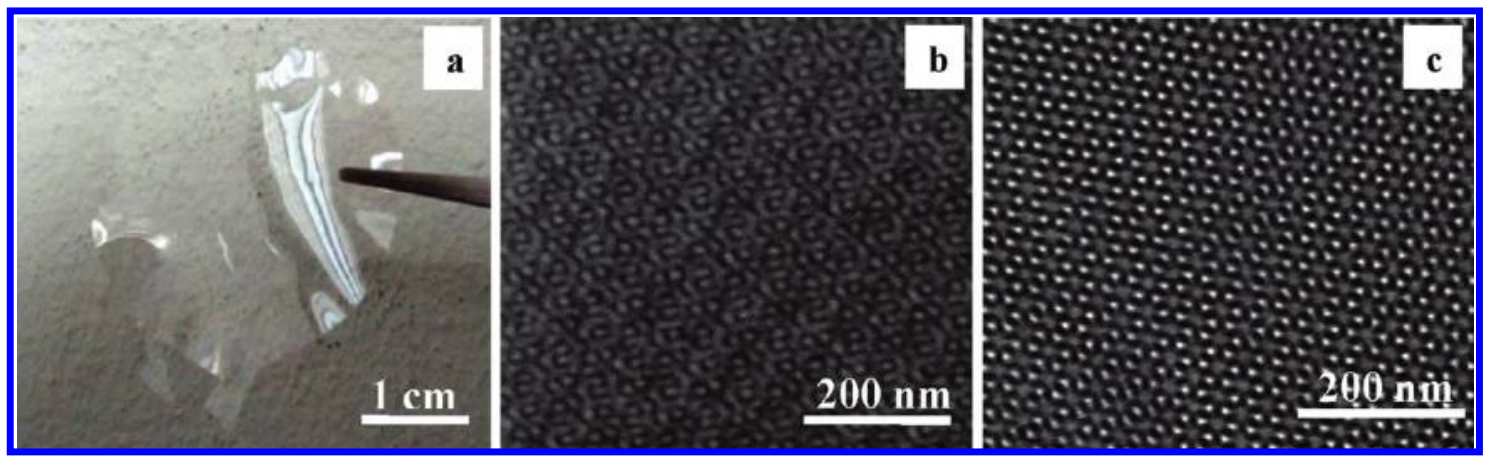

Figure 2. (a) Photograph of a nanoporous membrane; (b) SEM image of membrane's cross-section showing the (421) projection of gyroid morphology; (c) TEM micrograph of an ultrathin section of nanoporous membrane showing the (111) "wagon-wheel" projection of gyroid morphology.

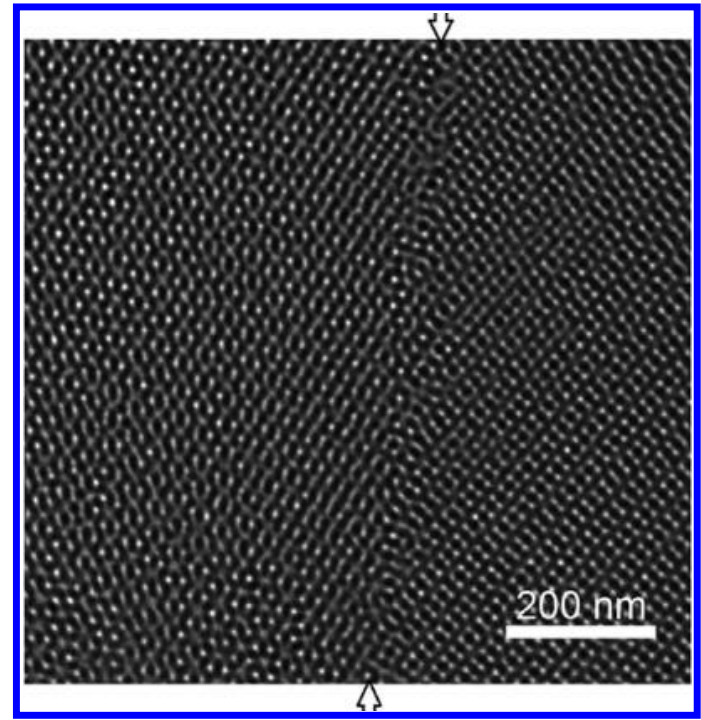

Figure 3. TEM micrograph of a gyroid grain showing a slightly tilted (111) wagon wheel projection on the left and a somewhat tilted (110) projection on the right of the grain boundary passing along the straight line joining the two arrow tips.

in Figure S3 of SI. A grain boundary zone is captured in the TEM image of Figure 3. There is substantial pore connectivity across the boundary, as judged from the perceived continuity of the white-gray shaded paths connecting the two grains. A similar conclusion can be reached by observing the TEM micrographs of two other grain boundary zones in Figure S3. Most of the pores on the images continue uninterrupted across the gyroid grain boundaries, which hints to the expectation that the mass transport is unaffected by the boundary zones. The membranes used in the present work are identical in the bulk morphology, porosity, pore size, and size distribution.

Surface Morphology. We used two different substrates, a glass plate and a fluorinated glass plate, to cast 1,2-PB- $b$-PDMS polymer solutions, thus, creating a distinct interfacial environment for the block copolymer films as-casted. For a limited number of samples, films were cast in glass Petri dishes and cross-linked with one surface free to nitrogen gas, which can be considered as a third substrate, in addition to the already mentioned glass and fluorinated glass substrates. During cross-linking, as the temperature increases from 20 to $140^{\circ} \mathrm{C}$, the microphase in the bulk (far away from the film surface) is expected to transform from lamellar (LAM) to gyroid (GYR) passing through the metastable hexagonally perforated layer morphology (HPL). ${ }^{17}$ LAM is the stable structure at room temperature and GYR at $140{ }^{\circ} \mathrm{C}$. Therefore, a gyroid structure was captured in the film bulk after cross-linking, as shown in Figures 2, 3, and S2a, and S3. However, the outer surface morphology of the block copolymer film depends also on the interfacial energy between polymer and the selected substrate. Accordingly, we investigated the outer surface of the resultant nanoporous membranes by SEM, contact angle, and XPS. Figure 4 is a collection of SEM images of the surface of nanoporous membranes prepared in contact with glass (Figure 4a) and in contact with fluorinated glass or nitrogen gas (Figures $4 b-f$ ). Apparently, neither of the surfaces shows the gyroid bulk morphology. Randomly distributed nanoporosity is observed on the surface contacting glass, with a pore size of $\sim 10 \mathrm{~nm}$ and a surface porosity of $35 \pm 10 \%$, as estimated by AFM image analysis (see Figure S2b of $\mathrm{SI})$. In contrast, the "nitrogen substrate" and the fluorinated substrate mostly produced a flat dense surface with no discernible pores as in the SEM image of Figures $4 \mathrm{~b}$,e. Figure $4 \mathrm{c}$ shows a top view of the edge of a nanoporous membrane prepared between the fluorinated substrates. A piece of "skin" with the same compact surface on the upper side as in Figure $4 b$ is partially peeled off from the surface. The surfaces beneath show characteristic features of HPL morphology, which is the transient morphology between LAM and GYR. ${ }^{17}$ Figure $4 \mathrm{c}$ evidences that the skin layer consists of sublayers, the outmost layer being LAM on the outer side and HPL on the inner side, followed by at least one additional HPL layer. A roughly $30 \mathrm{~nm}$ thick skin layer is also clearly observed in Figure $4 d$, which shows part of a cross section near the free 


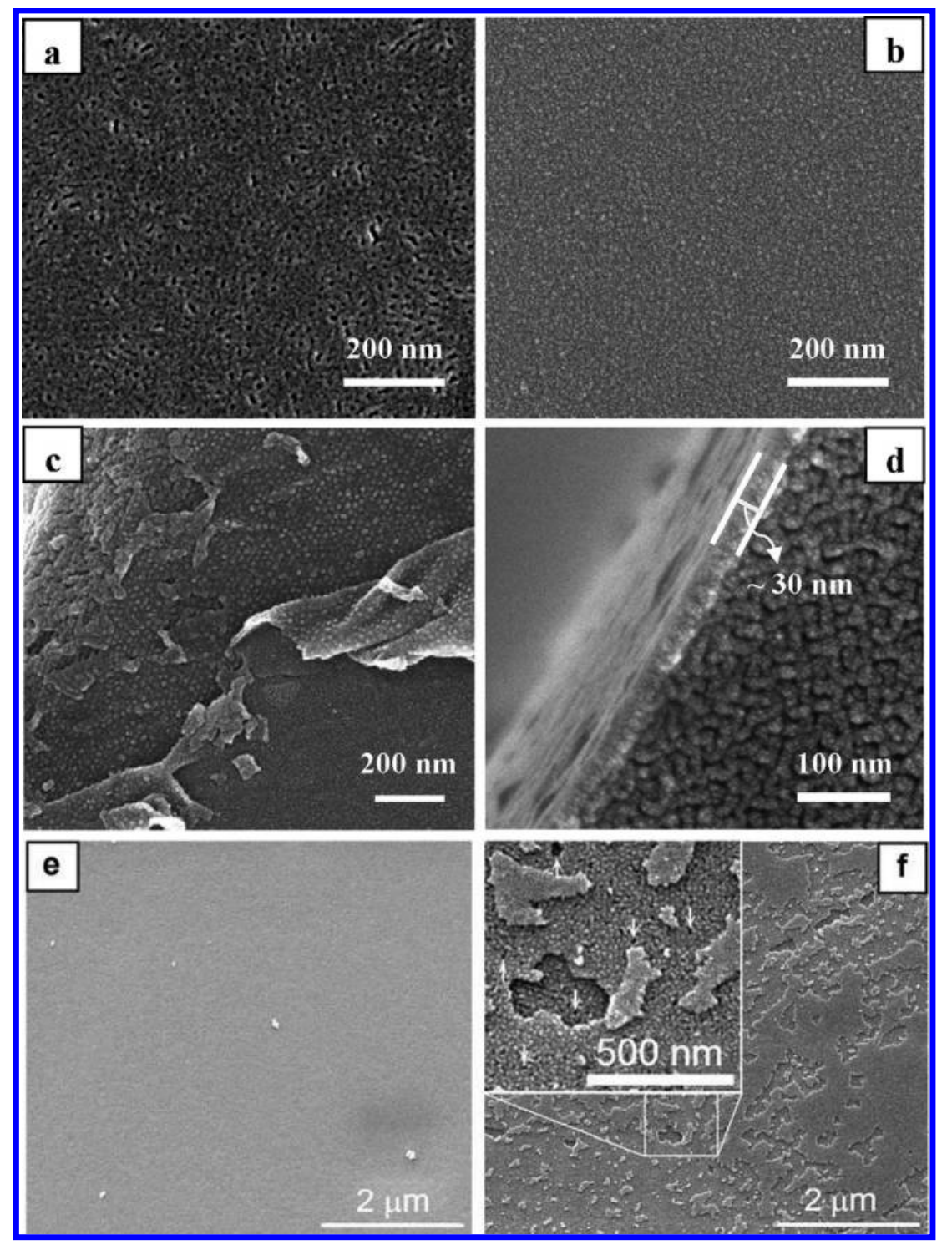

Figure 4. SEM images of nanoporous membranes' outer surface. (a) Surface in contact with glass (see also Figure S2b in SI for an AFM image of the same surface); (b, c, e, f) Surfaces in contact with fluorinated glass; (d) Cross-section near the free surface of a nanoporous film prepared by solvent casting. A portion of the skin layer of $30 \mathrm{~nm}$ thickness is highlighted; (e) Top view of skin region with intact upper "lamella" layer, and (f) with upper layer peeled off (same magnification as in (e)). The arrows in the zoom-inset of $(f)$ point to some of the holes that might percolate to the bulk porosity.

surface of a nanoporous film prepared by solventcasting. The periods of both LAM and HPL for a block copolymer sample similar to the precursor of this study are $\sim 21 \mathrm{~nm}$, as determined by SAXS. ${ }^{17}$ Therefore, the observed average skin layer thickness is equal to 1.5 periods of HPL ( $32 \mathrm{~nm}$ ) or to one PB lamella and one period of HPL (34 nm). Most of the skin surface is compact, like in Figures 4b,e. However, "peeled-off" areas, as seen in Figure $4 \mathrm{f}$ and Figures $\mathrm{S} 4 \mathrm{c}-\mathrm{f}$ are also observed randomly distributed throughout the skin area. At a larger magnification (inset of Figure 4f), a number of surface pores can be discerned within these areas; these pores might be connected to bulk pores, creating percolating defect clusters on the skin layer. This view is supported by the flow visualization experiments discussed further on in relation to Figure 7.
Hereafter, membranes prepared between two glasses are referred to as nonskin (ns) membranes, while membranes prepared between fluorinated glasses are referred to as double-skin (ds) membranes. Single-skin (ss) membranes are prepared between one fluorinated glass and one glass. SEM imaging was supplemented by advancing contact angle (CA) measurements on the surfaces of skin and nonskin membranes before and after removal of PDMS. The experimental data are summarized in the first data row of Table 1.

The CA value for the cross-linked double-skin membranes BD36-X-ds is similar to the reported value for pure PDMS. ${ }^{30}$ For the etched sample BD36-E-ds the CA value is close to that of the cross-linked 1,2-PB homopolymer (1,2-PB-H). Most likely only PDMS block segregates on the outmost surface of the BD36-X-ds 
TABLE 1. Experimental and Predicted Values of Advancing Contact Angle of Water on the Surface of Double-Skin and Nonskin Samples before and after PDMS Removal with Uncertainties as Subscripts

\begin{tabular}{|c|c|c|c|c|c|c|}
\hline & \multicolumn{2}{|c|}{ nonskin } & \multicolumn{2}{|c|}{ double-skin } & \multirow[b]{2}{*}{ PDMS } & \\
\hline & BD36-X-ns ${ }^{d}$ & BD36-E-ns ${ }^{d}$ & BD36-X-ds ${ }^{d}$ & $\mathrm{BD} 36-\mathrm{E}-\mathrm{ds} s^{d}$ & & $1,2-\mathrm{PB}-\mathrm{H}^{d}$ \\
\hline$\theta_{\exp }^{a}\left(^{\circ}\right)$ & $91.1_{3.5}$ & 107.1 $1_{9.7}$ & $105.6_{0.6}$ & $93.3_{4.1}$ & $106_{6}{ }^{c}$ & $91.0_{0.8}$ \\
\hline$\theta_{\mathrm{cal}}^{b}\left(^{\circ}\right)$ & $91.0_{0.8}$ or $96.1_{2.9}$ & $111.2_{6.0}$ & $106_{6}$ & $91.0_{0.8}$ & & \\
\hline
\end{tabular}

${ }^{a}$ Experimental values of advancing contact angle of water. ${ }^{b}$ Calculated values of water contact angle based on the observations from SEM, using eq 1. 'Value of water contact angle on PDMS surface reported in ref 30. ${ }^{d}$ BD36-X-ns: cross-linked nonskin; BD36-E-ns: etched nonskin; BD36-X-ds: cross-linked double skin; BD36-Eds: etched double skin samples; 1,2-PB-H: cross-linked 1,2-PB homopolymer.

sample; accordingly, a 1,2-PB layer fully covers the outermost surface of the BD36-E-ds sample after PDMS removal. For the ns membranes, the water contact angle increased from the cross-linked sample BD36-X$\mathrm{ns}$ to the etched sample BD36-E-ns. This might be due to the porous surface (Figures $4 a$ and $S 2 b$ ) of the etched sample where air-filled nanopores lower the surface energy. We compared the obtained CA values with predictions by Cassie's equation, ${ }^{31}$ using the information of surface porosity from the AFM images (see Figure S2b).

$$
\cos \theta_{p}=f_{\mathrm{A}} \cos \theta_{\mathrm{A}}+f_{\mathrm{B}} \cos \theta_{\mathrm{B}}
$$

where $\theta_{p}$ is the water contact angle of the surface made of $A$ and $B . f_{\mathrm{A}}$ and $f_{\mathrm{B}}$ are surface fractions of component $A$ and $B$, respectively. In our case, $A$ is 1,2$\mathrm{PB}$ and $\mathrm{B}$ is either PDMS for the cross-linked samples or air for the etched samples. The calculated values are given in the second data row of Table 1. For the BD36$X$-ns sample, there are two possibilities, either (1) the outmost surface is covered by a layer of pure 1,2-PB, then the calculated $C A$ value is $91.0^{\circ}$; or (2) a CA value of $96.1 \pm 2.9^{\circ}$ can be calculated assuming coexistence of 1,2-PB and PDMS on the surface with the same concentration of PDMS as the surface porosity of the etched sample ( $35 \pm 10 \%$ PDMS). XPS analysis favors this last scenario (see Supporting Information). The reason for the lower observed $C A$ value than the $96.1^{\circ}$ of scenario (2) may be due to some slight surface oxidation present on the outermost PB layer, accidentally lowering the overall contact angle close to that of pure 1,2-PB. After PDMS removal, the BD36-E-ns sample shows a porous surface with surface porosity of $35 \pm 10 \%$ (Figures $4 a$ and S2b), consistent with a calculated CA value of $111.2 \pm 6.0^{\circ}$. For the BD36-Xds sample, the observed value is consistent with a surface fully covered by PDMS $\left(106^{\circ}\right) .^{30}$ The enrichment with PDMS of the surface in contact with fluorinated glass (or of the free surface) is driven by minimization of interfacial energy. A CA value of $91^{\circ}$, equal to that of pure 1,2-PB, is predicted for the BD36-
E-ds sample. This expectation is based on the observation of nonporous surface by the SEM images in Figures $4 \mathrm{~b}$,e combined with the quantitative removal of PDMS in the etching process. ${ }^{17}$ The surface composition of the skin and nonskin samples was further assessed by XPS (see Table S1 in SI). A short discussion of the XPS results and their consistency with the results from the other techniques is presented in the SI. An overall picture emerges from the results of surface characterization. The high surface energy substrate (glass) does not seem to show preference for any of the blocks; a homogeneously porous surface is observed after PDMS etching, although without the characteristic regularity of the GYR morphology. The low surface energy substrates (nitrogen gas or fluorinated glass) stabilize the LAM microstructure at the polymer interface by promoting the enrichment with PDMS of the polymer surface in contact with the substrate. This happens even at the cross-linking temperature of $140{ }^{\circ} \mathrm{C}$ where GYR is the thermodynamically stable microstructure in the polymer bulk. After PDMS etching, a $\sim 30 \mathrm{~nm}$ thick skin layer of cross-linked 1,2-PB is observed at the surface, showing a smooth compact outer side, compatible with the LAM morphology, and beneath, a microstructure compatible with HPL. Sporadic "islets" extending from few to few tens of micrometers are observed, where the outer compact layer is peeled off and the HPL structure exposed; the HPL layer in these areas is randomly perforated by pores that possibly percolate into the bulk (see Figures $4 \mathrm{f}$ and $\mathrm{S} 4 \mathrm{e}, \mathrm{f}$ ). We speculate that the peeled-off areas form at places with high abundance of porous defects on the underlying HPL. The higher is the density of defects, the weaker becomes the connectivity of the upper layer to the material beneath, increasing its susceptibility to stresses generated by crosslinking and etching. The porous defects themselves might occur due to some degree of coexistence (or structural frustration) between the stable GYR in the bulk and the metastable HPL. Such a coexistence has been earlier observed in the bulk of a similar block copolymer by SAXS measurements at different temperatures. ${ }^{17}$ The length-scale of the peeled-off areas is probably related to external factors, such as variations in thermal contact between the polymer and the substrate during the cross-linking reaction. These may cause disparity in cross-linking rates and therefore variations in the morphology at the polymer-substrate interface before it becomes fully frozen due to the high degree of cross-linking.

Membrane Performance. Diffusive Permeability. Glucose was chosen as a solute for studying the permeation of the nanoporous membranes. Results on glucose diffusion through $20 \mu \mathrm{m}$ thick nonskin (ns), single-skin (ss) and double-skin (ds) membranes are presented in Figure 5. Figure $5 \mathrm{a}$ is a plot of glucose concentration in the receiver cell as a function of time. As expected, the 


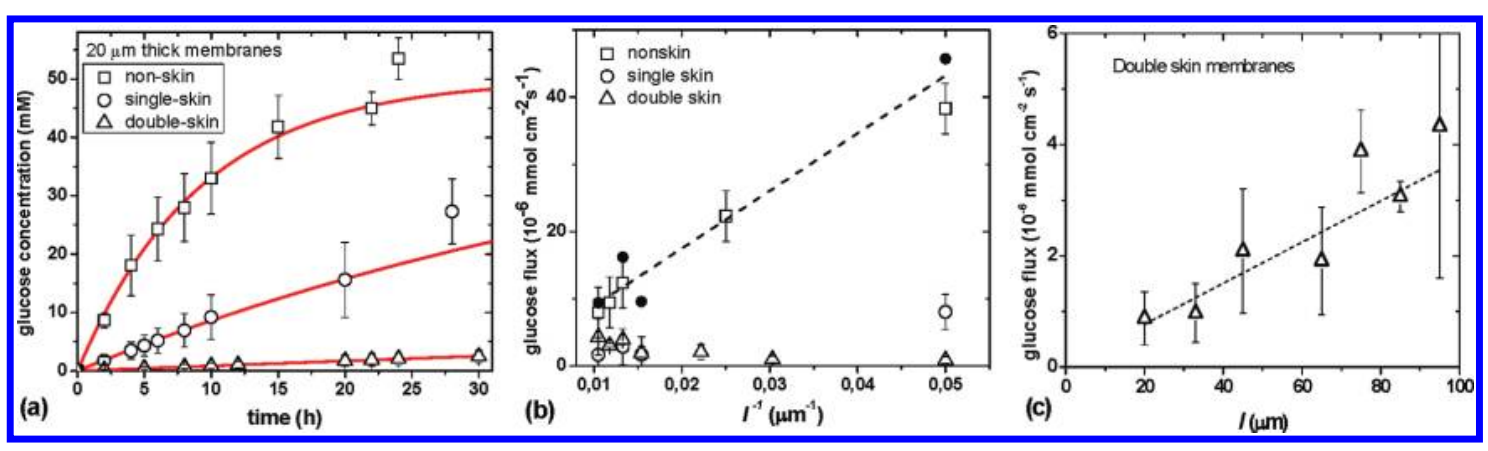

Figure 5. Glucose diffusion across nonskin, single-skin and double-skin nanoporous membranes. (a) Glucose concentration in the receiver cell as a function of time for $20 \mu \mathrm{m}$ thick membranes. The solid lines are best fits by eq 2 to the three sets of data (excluding the two data points at longest times for ns and ss, which show higher concentrations, probably due to some accidental water evaporation); (b) glucose permeation vs reciprocal of membrane thickness. The filled circles are the permeability data for the ss membrane multiplied by $5.7\left(=D_{\mathrm{ns}} / D_{\mathrm{ss}}\right.$ calculated from the best fits in Figure $\left.5 \mathrm{a}\right)$. The dashed line is a linear fit to the data (squares and solid circles); (c) glucose flux through ds membranes as a function of membrane thickness. The flux increases with membrane thickness!.

ns membrane exhibits the fastest glucose permeation, reaching equilibrium within $30 \mathrm{~h}$. The ds membrane shows the slowest permeation ascribed to the big resistance of the skin layer on both sides of the membrane. The equilibrium state is not achieved for ss and ds membranes within the measurement time window. Figure $5 \mathrm{~b}$ shows the dependence of the initial glucose permeation rate on the inverse of membrane thickness $I^{-1}$ for the ns, ss, and ds samples. The glucose diffusion flux values across the ns membranes (open squares) can be described by a simple linear dependence on the inverse membrane thickness. The flux values through the ss membranes (open circles) show a similar dependence but with a significantly reduced slope. The filled circles in Figure $5 \mathrm{~b}$ are the flux data for the ss membrane multiplied by the factor of effective diffusion coefficients calculated from the permeability data through the $20 \mu \mathrm{m}$ thick samples of Figure $5 \mathrm{a}$ (and eq 2 below). Figure $5 c$ shows the permeability data for the ds membranes of Figure $5 b$ replotted against membrane thickness $I$, instead of $I^{-1}$. The surprising flux increase with membrane thickness discerned in Figure $5 c$ can be understood from the model of Figure 6 presented below.

The effective membrane diffusion coefficient $D_{e}$ $\left(\mathrm{cm}^{2} / \mathrm{s}\right)$ of glucose in water can be determined by the following mass balance equation:

$$
\ln \frac{c_{0}}{c_{1}(t)-c_{2}(t)}=\beta \frac{D_{e}}{l} t, \quad \beta=A_{0}\left(\frac{1}{V_{1}}+\frac{1}{V_{2}}\right)
$$

where $c_{0}$ is the initial glucose concentration in the donor cell; $c_{1}(t)$ and $c_{2}(t)$ are the glucose concentrations at time $t(\mathrm{~s})$ in the donor and receiver cells, respectively; $c_{2}(0)=0 ; V_{1}$ and $V_{2}$ are the solution volumes $\left(\mathrm{cm}^{3}\right)$ in the two cells $\left(V_{1}=V_{2}\right) ; l$ is the thickness $(\mathrm{cm})$, and $A_{0}$ is the area $\left(\mathrm{cm}^{2}\right)$ of the membrane exposed to the solution. We assume the external mass transfer boundary layer resistance on each side to be minimized by stirring, and that there is no mass accumulation

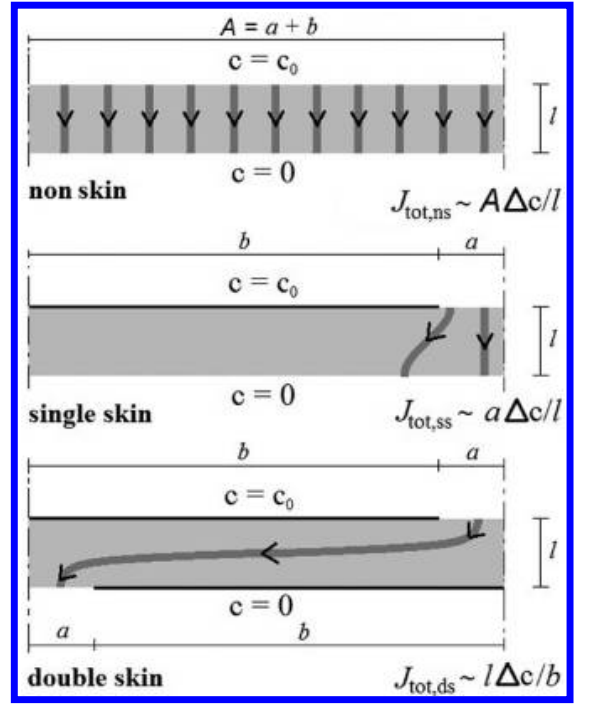

Figure 6. Proposed model interpreting the flow through non skin, single skin and double skin membranes observed in Figure 5. The membranes are modeled as sheets of thickness I (gray areas) and the skin layer as an infinitely thin impermeable film (black lines) with defects. Defects are modeled as parallel non skin stripes of width $2 a$ and regular spacing $2 A=2(b+a)$. Cross sections of only the symmetry units of the model are shown. In the case of double skin membrane the bottom skin layer is the same as the upper skin layer, but with defect stripes parallelly displaced by $A$. The dark gray lines with arrows schematically show diffusion transport lines for glucose. The concentrations at $t=0$ are shown. The proportionality relations for the total fluxes $J_{\text {tot,ss }}$ and $J_{\text {tot,ds }}$ are expected to be valid at the limiting case of $l / b \ll 1, a / b \ll 1$, and $I / 2 a \ll 1$.

inside the membrane. The solid lines are best fits to the data of Figure $5 \mathrm{a}$ by eq 2 . The fits are very good except for the two data points exceeding $20 \mathrm{~h}$ for the $\mathrm{ns}$ and ss membranes, where we believe that the raised concentration of glucose is caused by some accidental water evaporation. From the fits the effective diffusion coefficient of glucose was calculated for each type of $20 \mu \mathrm{m}$ thick membrane: $D_{\mathrm{ns}}=1.20 \times 10^{-6} \mathrm{~cm}^{2} / \mathrm{s} ; D_{\mathrm{ss}}=2.09 \times$ $10^{-7} \mathrm{~cm}^{2} / \mathrm{s} ; D_{\mathrm{ds}}=1.88 \times 10^{-8} \mathrm{~cm}^{2} / \mathrm{s}$. The total diffusive fluxes of glucose for the three types of membranes are 
proportional to the respective effective diffusion coefficients: $J_{\text {tot,ns }}=5.7 \times J_{\text {tot }, \text { ss }}=64 \times J_{\text {tot,ds }}$. The flux through ds membranes is almost 2 orders of magnitude lower than through ns membranes.

Conceptual Models. The skin morphology on ss and ds membranes is expected to be the same, due to the identical procedure of fabrication. Therefore a naive model of additive resistance to flux from skin layer(s) and bulk would predict a maximum reduction by a factor of 2 for the total flux through the ds relative to the ss case. The value is significantly smaller than the experimental reduction factor of 11. This strong reduction of flux from ns to ss to ds membranes might be due to the existence of few, rather big defects randomly distributed on the skin layer(s) with mean interdistance $2 A$ much bigger than both the membrane thickness $I$ and the defect size $2 a$, as modeled in Figure 6. A single skin membrane with cracks was modeled similarly in ref 32 . The flux proportionality relations on the geometric parameters shown in Figure 6 are expected to be valid at the limiting case of, $I / b \ll 1, a / A \approx a / b \ll 1$ and $I / 2 a \ll 1$. The model of Figure 6 reduces the diffusion transport into a twodimensional problem. Despite this simplification we believe that it captures the physics behind the observed differences of diffusive fluxes for the three types of membranes. Notice that $J_{\text {tot,ds }}$ is expected to increase with the membrane thickness $I$, in accordance with the data of Figure $5 c$. Such an unintuitive behavior is due to the skin boundary conditions in the ds membranes imposing a dominant radial mass transport (parallel to membrane surface). The third condition $(/ / 2 a \ll 1)$ is not necessary for this to happen.

As for the ss samples, the model predicts a flux proportional to the defect area and inversely proportional to membrane thickness. This is actually true when the defect size is much larger than the sample thickness (third condition: $2 a \gg 1$ ); in that case, the flux at the defect edges gives a negligible contribution to the total flux through the defect opening. Otherwise the flux ratio $J_{\text {tot,ss }} / J_{\text {tot,ns }}$ will exceed the defect fractional area, due to the edge contribution. ${ }^{32}$ In the mentioned limiting case the flux is reduced by a factor of $a / A$ relative to the flux through the ns membrane, which is equal to the fractional area of defects in ss. Equalizing this factor to the experimental flux reduction factor of $5.7\left(=D_{\mathrm{ns}} / D_{\mathrm{ss}}\right)$, would correspond to a fractional area of defects in the model equal to $17 \%$. From the above discussion $17 \%$ is an upper limit for the fractional area of defects; in cases where the flux around the defect edges is significant the actual fractional area of defects will be less than $17 \%$. With the above interpretation, values of the model geometrical parameters reproducing the experimental observations for $I=20 \mu \mathrm{m}$ are $A=180 \mu \mathrm{m}$ and $a=30 \mu \mathrm{m}$ (or more accurately $a \leq 30 \mu \mathrm{m}$ ). The "fit" model values $I / b=0.13, a / b \leq 0.20$ and $I / 2 a \approx 0.33$ only "mildly" satisfy the limiting model conditions $(/ / b \ll 1, a / b \ll 1$, and $I / 2 a \ll 1$ ); this is especially the case for the third condition, which means that the edge flux cannot be neglected. At diminishing thickness, / the first and third limiting model conditions are increasingly approached. Compared to the essentially 1-D skin layer model of Figure 6 the edge flow contribution is expected to be more important in a more realistic 2-D skin layer with, for example, circular defects. This means that the expected defect fractional area in the case of real membranes might be well below the above estimated $17 \%$ value. In spite of the simplicity of the model its predictions are quite realistic, as shown in the coming paragraph on "Flow Visualization".

Before that we briefly mention another model showing a nonlinear flux decline from nonskin to single-skin to double-skin membranes (see Figure S5 of SI). This is the case of membranes composed of cylindrical pores aligned normal to the membrane surface. Like in the first model, the membrane material is impermeable to the solute. In such case the flux ratio $J_{\mathrm{ds}} / J_{\mathrm{ss}} / J_{\mathrm{ns}}$ is expected to equal $\alpha^{2} / \alpha / 1$, where $\alpha$ is the surface fraction of the randomly distributed defects on the skin surface. Thus, for $\alpha=0.1$ the flux through ds membranes is expected to be only $1 \%$ of the flux through ns membranes. At a defect surface fraction $\alpha=0.125$ a ratio $J_{\text {ds }} / J_{\text {ss }} / J_{\text {ns }}=1: 8: 64$ is predicted, which is close to the experimental values from Figure 5 . In contrast to the model of Figure 6, the cylindrical pore model of Figure S5 (1) does not allow flux increase with membrane thickness and (2) imposes a laterally nonspreading diffusion profile for the solute. Neither feature is supported experimentally, as seen in Figure $5 c$ relative to feature (1), and as will be shown in the next paragraph relative to feature (2). From the structural viewpoint the cylindrical pore model is probably not relevant for the gyroid morphology, where the isotropic pore percolation within each gyroid grain and the observed high pore connectivity through the grain boundaries render quite unlikely any kind of preferential directional diffusion transport through the membrane bulk. However, in our opinion, it is a heuristically interesting model, it can be generalized to accommodate isotropic flow by dropping the pore alignment condition, and most importantly, it may be relevant for porous media with other morphologies.

Flow Visualization. In a tentative to test the predictions from the simple models of Figures 6 and S5, one nonskin and a number of skin membrane samples were exposed to a Rhodamine 6G (R6G) solution in ethanol-water, as described in the SI. Notice that for practical reasons skin films of thickness $200 \mu \mathrm{m}$ were used, which exceeds the thickness values of the membranes used in relation to Figure 5.

Figure 7 shows fluorescence pictures of fracture surfaces from one nonskin membrane, exposed for $1 \mathrm{~s}$ to R6G and one skin membrane, exposed for $10 \mathrm{~s}$ and 


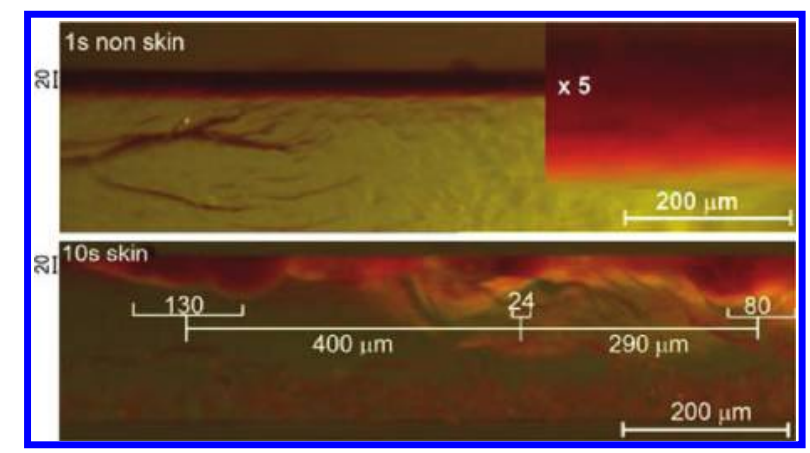

Figure 7. Optical fluorescence microscopy pictures of cross sections of two gyroid nanoporous films, the upper one with a non skin top surface and the bottom one with a skin top surface, exposed for, respectively, 1 and $10 \mathrm{~s}$ to a R6G solution. The R6G in the nanopores appears as a dark red layer on the nonskin membrane or as red "blobs" on the skin membrane. The less intensely colored regions at the upper surface of the skin membrane are probably caused by R6G "blobs" deeper in the sample. The red and yellow irregular striations are reflections of the intense R6G fluorescence red light by the uneven fracture surfaces. A 5 times magnified image of the top layer is shown as an inset, highlighting the horizontal homogeneity of the R6G diffusion layer through the non skin membrane. The distances in $\mu \mathrm{m}$ show approximate sizes of skin defect clusters and distances among the neighbor defect clusters at the cross-section of the skin sample. The vertical side bars of $20 \mu \mathrm{m}$ show the film thickness used in the permeability experiments of Figure 5a. For more details, see SI and Figure S6.

then dried. The visual difference between the "frozen" flow patterns in the two membranes is striking! The diffusion front of R6G through the nonskin sample is parallel to the surface and agrees perfectly with the expectations of both non skin membrane models in Figures 6 and S5. However, only the model of Figure 6 is supported by the observed advancing front through the skin sample. The diffusion front of R6G in the skin sample is very irregular, with three separate advancing fronts, 290 and $400 \mu \mathrm{m}$ apart, visible in the picture. The rounded profile of the R6G front testifies for an isotropic diffusion in the bulk and for rather well localized "sources" (defects) on the skin. Pictures in Figure S6 taken after 1, 5, 10, and $60 \mathrm{~s}$ of contact times with the R6G solution show that:(1) local diffusion blobs are distributed into clusters; (2) depending on the contact time the distance between separate diffusing blobs varies between few $\mu \mathrm{m}$ and $500 \mu \mathrm{m}$, with the bigger clusters spacing in the range of $200-500 \mu \mathrm{m}$; (3) the diffusing fronts from the local skin defect sources merge laterally at longer diffusion times creating more or less flat fronts advancing into the bulk; (4) at $60 \mathrm{~s}$ of contact time R6G fills most of the porous film volume and lateral diffusive spreading imposed by the opposite film surface is observed. The scenario that emerges from the flow visualization experiments on the skin samples is that the solute diffuses into the bulk from clusters of localized surface pores. The typical cluster size is small relative to their average separation; this last is big relative to the membrane thicknesses of Figure 5 (and comparable to the separation calculated by the model, $360 \mu \mathrm{m}$ ), just like the model of Figure 6 wants it! The merger of diffusion fronts from neighboring defects creates effective flow profiles that in crosssection resemble those expected from the model of Figure 6. However, the observed profiles testify that the defect-edge flow cannot be neglected in the skin membranes, unless the membrane thickness is very

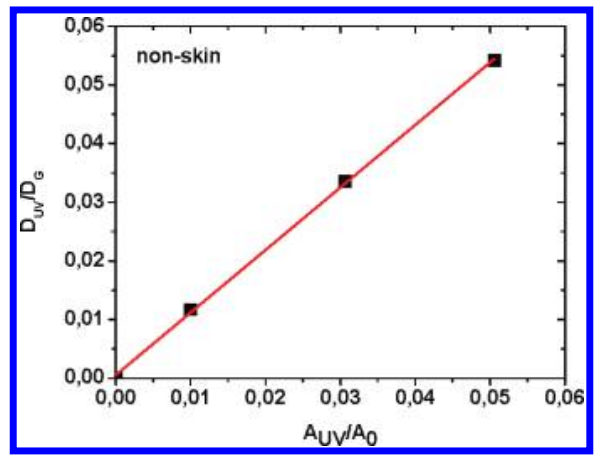

Figure 8. Ratio of effective diffusion coefficient of glucose through the selectively hydrophilized membranes $D_{U v}$ relative to that of glucose transport in the fully hydrophilized membranes $D_{\mathrm{G}}$ relative to the ratio of UV-irradiated fractional surface area $A_{\cup v} / A_{0}$.

much reduced. From the discussion following Figure 6 this means that the fractional area of defects in the model reproducing the experimental flux values would be less than 0.17 . The defect clusters on real membranes are most probably related to the distribution and size of the peeled-off areas and pores shown by the SEM images of Figures $4 \mathrm{f}$ and S4c- $\mathrm{f}$. An accurate assessment of the origin, size, and distribution of defect clusters on skin membranes is yet to be done by combining flow visualization and SEM analyses on a large number of samples.

Controlled Active Porosity. Throughout the rest of the article we focus on nonskin membranes. The effective diffusion coefficient $D_{e}$ across a porous film can be related to the diffusion coefficient in free solution $D_{0}$ by the following equation: ${ }^{33}$

$$
D_{e}=\frac{\varepsilon \delta D_{0}}{\tau}
$$

where $\tau$ is tortuosity, $\varepsilon$ is porosity, and $\delta$ is constrictivity of solute through a pore. We created a series of nanoporous membranes with varying active porosity 
as a means to tune the glucose permeation. We previously reported that the originally hydrophobic membranes can be turned into hydrophilic via UV photooxidation ${ }^{20}$ or thiol-ene photochemistry. ${ }^{21}$ Water can spontaneously and exclusively fill the hydrophilized nanopores. As a result, the hydrophilized nanopores are permeable to the diffusing species in aqueous solutions, while no diffusion happens in the unmodified pores. This provides an easy means to change active porosity by hydrophilizing selective regions of the membrane. Figure 8 nicely shows that the effective diffusion coefficient of glucose in water is proportional to the active porosity of the membrane expressed in terms of the fractional surface area exposed to UV.

The results above demonstrate that the permeation rate of glucose through nanoporous cross-linked 1,2PB membranes can be widely varied either by manipulating the surface morphology of the membrane or by changing the active porosity.

Membrane Selectivity. Selectivity was studied as the second important characteristics of the membrane performance. A series of molecules were used to evaluate the selectivity of $20 \mu \mathrm{m}$ thick nonskin nanoporous membranes including antibiotics, proteins and other biomolecules, as listed in Table 2. It summarizes the basic characteristics of the tested solutes. The values of effective diffusion coefficient derived from eq 3 are given in the next-to-last column of the table. In the case of proteins, there was a time lag for adsorption equilibration. This equilibration period ensured that the concentration profile across the membrane eventually reached a quasi-steady-state. ${ }^{34}$ Therefore, in the case of proteins the starting time for a linear fitting was taken after equilibration rather than at $t=0$. The values of selectivity $\alpha$, defined as the ratio of effective diffusion coefficient of glucose to that of a given solute are summarized in the last column of Table 2 . We found that the $D_{e}$ values for ciprofloxacin (3), vancomycin (5), cytochrome C (6), and myoglobin (7) were significantly lower than expected from their sizes (see below). Myoglobin and albumin showed extremely little or no permeation so that the concentration on the receiver cell was lower than the detection limit of the UV-visible spectrometer used. See also Table S3 for the molecular structures of the solutes.

In general, solute transport in a constricted pore reduces mainly due to equilibrium partitioning and hydrodynamic effects. ${ }^{43}$ The constrictivity can be calculated by

$$
\delta=D_{e} \tau / D_{0} \varepsilon
$$

where tortuosity for the gyroid porosity is taken $\tau=$ $1.5 i^{44}$ porosity $\varepsilon=0.4$ is taken equal to the volume fraction of etched PDMS; and the experimental $D_{e}$ values are listed in Table 2. To characterize the hindered diffusion through the nanoporous membranes, the constrictivity $\delta$ is plotted $v s$ the ratio of hydrodynamic
TABLE 2. Summary of Effective Diffusion Coefficient Selectivity, and Related Parameters of the Solutes Tested in the Nanoporous Membranes ${ }^{a}$

\begin{tabular}{|c|c|c|c|c|c|c|}
\hline No. & solute & $\begin{array}{c}M_{\mathrm{w}} \\
(\mathrm{g} / \mathrm{mol})\end{array}$ & $\begin{array}{c}R_{\mathrm{s}}^{b} \\
(\mathrm{~nm})\end{array}$ & $\begin{array}{c}D_{0}^{b} \\
\left(\mathrm{~cm}^{2} / \mathrm{s}\right)\end{array}$ & $\begin{array}{c}D_{e} \\
\left(\mathrm{~cm}^{2} / \mathrm{s}\right)\end{array}$ & $\alpha_{\text {glucose } / i}$ \\
\hline 1 & hydrogen peroxide & 34.0 & 0.16 & $1.30 \times 10^{-5}$ & $3.20 \times 10^{-6}$ & 0.40 \\
\hline 2 & glucose & 180.2 & 0.32 & $6.73 \times 10^{-6}$ & $1.28 \times 10^{-6}$ & 1.00 \\
\hline 3 & ciprofloxacin $\cdot \mathrm{HCl} \cdot \mathrm{H}_{2} \mathrm{O}$ & 385.8 & 0.55 & $4.00 \times 10^{-6}$ & $5.50 \times 10^{-8}$ & 23 \\
\hline 4 & ampicillin $\mathrm{Na}$ salt & 371.4 & 0.58 & $3.75 \times 10^{-6}$ & $5.42 \times 10^{-7}$ & 2.36 \\
\hline 5 & vancomycin $\cdot \mathrm{HCl}$ & 1485.7 & 0.79 & $3.64 \times 10^{-6}$ & $3.40 \times 10^{-8}$ & 37 \\
\hline 6 & cytochrome C & 12500 & 1.46 & $1.50 \times 10^{-6}$ & $3.40 \times 10^{-9}$ & 376.47 \\
\hline 7 & myoglobin ${ }^{d}$ & 16700 & 2.33 & $9.38 \times 10^{-7}$ & $<D L$ & $<D L$ \\
\hline 8 & albumin $^{d}$ & 66000 & 3.60 & $6.07 \times 10^{-7}$ & $<D L$ & $<D L$ \\
\hline
\end{tabular}

${ }^{a}$ The description of experimental conditions is found in the Methods section. ${ }^{b}$ Hydrodynamic radius of the solute $R_{s}$ was calculated from the diffusion coefficient in free solution $D_{0}$, using Stokes - Einstein equation. $D_{0}$ are literature values. ${ }^{35-42}$. 'Selectivity $\alpha_{\text {glucose/i }}$ is defined as the effective diffusion coefficient of glucose relative to that of the solute tested. ${ }^{d}$ Below detection limit (DL; $10 \mu \mathrm{g} / \mathrm{mL}$ for myoglobin and $50 \mu \mathrm{g} / \mathrm{mL}$ for albumin).

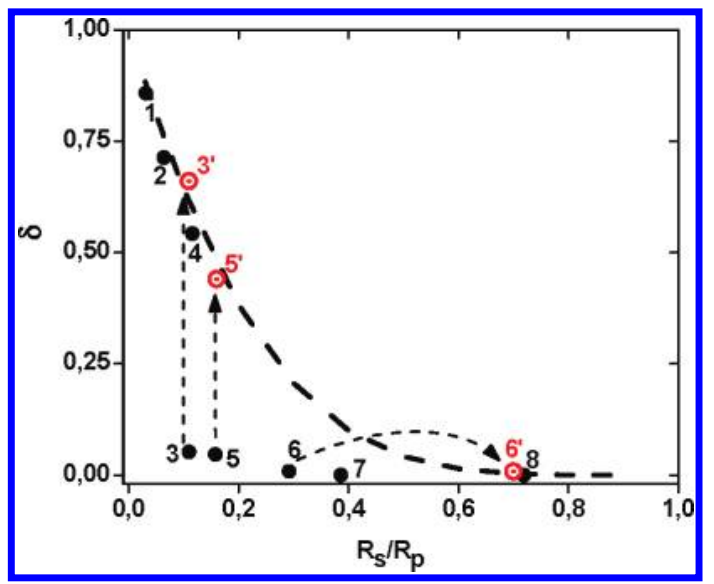

Figure 9. Plot of solutes' constrictivity as a function of the ratio between solute hydrodynamic radius and pore radius. The experimental conditions for the data denoted by the solid circles are described in the Methods section. The detailed explanation of the data marked by $3^{\prime}$ and $5^{\prime}$ and shown by open circles is given in relation to the discussion of Figure 10. The data point $6^{\prime}$ is explained in the main text. The bold dashed curve is the prediction from the Bungay and Brenner model (see Supporting Information). The vertical coordinate for samples $\mathbf{7}$ and $\mathbf{8}$ is zero, as explained in footnote $d$ of Table 2 .

radius of solutes to pore radius $R_{\mathrm{s}} / R_{\mathrm{p}}$ in Figure 9 . Here the pore radius $R_{\mathrm{p}}=5.0 \mathrm{~nm}$ is determined by gas diffusion and water flux measurements, consistent with the estimates from TEM, SEM, and AFM; it is lower than the pore radius determined by nitrogen adsorption. ${ }^{45}$ The hydrodynamic radius $R_{\mathrm{s}}$ of the solutes (Table 2) was calculated from the diffusion coefficient in free solution $D_{0}$ using the Stokes-Einstein equation. The plot reveals that space confinement and other interactions in the nanopores significantly affect the diffusivity of the solutes, as discussed below.

The experimentally derived constrictivity values are compared with the prediction from the Bungay and Brenner model ${ }^{46}(\mathrm{BB})$ shown by the bold dashed line in 
TABLE 3. Basic Information on Experimental Conditions for the Diffusion Tests

\begin{tabular}{|c|c|c|c|c|}
\hline No. & solute & concentration & solvent & $\begin{array}{c}\text { UV absorbance } \\
\text { peak (nm) }\end{array}$ \\
\hline 1 & hydrogen peroxide & $5 \mathrm{mM}$ & $\begin{array}{c}10 \mathrm{mM} \text { imidazole } \\
\text { buffer pH } 6.5\end{array}$ & \\
\hline 2 & glucose & $100 \mathrm{mM}$ & $\mathrm{Dl}^{a}$ & \\
\hline 3 & ciprofloxacin $\cdot \mathrm{HCl} \cdot \mathrm{H}_{2} \mathrm{O}$ & $\begin{array}{l}0.02 \mathrm{~g} / \mathrm{L} \\
1 \mathrm{~g} / \mathrm{L}\end{array}$ & $\begin{array}{l}\mathrm{PBS}^{b} \text { buffer } \mathrm{pH} 7.4 \\
\text { DI }\end{array}$ & 278 \\
\hline 4 & ampicillin Na salt & $1 \mathrm{~g} / \mathrm{L}$ & DI & 257 \\
\hline 5 & vancomycin $\cdot \mathrm{HCl}$ & $\begin{array}{l}0.2 \mathrm{~g} / \mathrm{L} \\
1 \mathrm{~g} / \mathrm{L}\end{array}$ & $\begin{array}{l}\mathrm{DI} \\
\mathrm{DI}\end{array}$ & 281 \\
\hline 6 & cytochrome $C^{c}$ & $1 \mathrm{~g} / \mathrm{L}$ & PBS buffer pH 7.4 & 410 \\
\hline 7 & myoglobin ${ }^{c}$ & $1 \mathrm{~g} / \mathrm{L}$ & PBS buffer pH 7.4 & 410 \\
\hline 8 & Aabumin ${ }^{c}$ & $1 \mathrm{~g} / \mathrm{L}$ & PBS buffer pH 7.4 & 278 \\
\hline
\end{tabular}

${ }^{a}$ The deionized water $\mathrm{DI}$ was used as it is; $\mathrm{pH}=4.5$. ${ }^{b} \mathrm{PBS}$ (phosphate buffered saline) buffer consists of $0.01 \mathrm{M}$ phosphate buffer, $0.0027 \mathrm{M}$ potassium chloride, and $0.137 \mathrm{M}$ sodium chloride with $\mathrm{pH}=7.4$. $^{\mathrm{C}}$ The diffusion tests of all the proteins were performed at $8^{\circ} \mathrm{C}$; the diffusion tests of the other molecules were run at room temperature.

Figure 9. The BB model describes quite accurately diffusion of rigid molecules through a cylindrical nanopore for $R_{\mathrm{s}} / R_{\mathrm{p}}<1$; the model considers only steric effects and hydrodynamic interactions between solute and pore wall. It is evident from Figure 9 that hydrogen peroxide, glucose and ampicillin follow the model prediction. This indicates that at the measurement conditions summarized in Table 3 of the Methods section, size exclusion and hydrodynamic interactions govern the diffusion of these three molecules in the nanopores. The first two do not bear electrical charges, while ampicillin $\left(\mathrm{p} K_{\mathrm{a} 1}=2.5\right.$ and $\left.\mathrm{p} K_{\mathrm{a} 2}=7.3\right)$ is a zwitterion at $\mathrm{pH} \approx 4.5$. On the other hand, the hindered diffusion of the other molecules is much slower and the selectivity much higher than expected by the BB equation. The permeation of solutes across a membrane does not only depend on the size of solutes or pores; in most cases, it strongly depends on a number of solute-solute and solute-membrane interactions, ${ }^{34,46-49}$ such as electrostatic, hydrophobic, charge transfer, and hydrogen bonding interactions. These interactions can be significantly influenced by physicochemical parameters, such as $\mathrm{pH}$, ionic strength, transmembrane pressure, surface chemistry of the nanopore wall, and so on. We could not investigate in any systematic fashion the complex parameter space of such interactions for the solutes of the present study. Even so, we show through the rest of the section that (1) at changed conditions, ciprofloxacin and vancomycin exhibit constrictivity values close to the BB prediction of Figure 9; (2) the large deviations observed in Figure 9 for the constrictivity values of proteins relative to the $\mathrm{BB}$ prediction may be due to protein adsorption effects, vastly reported in the literature.

Starting with proteins, the significant reduction in diffusion of cytochrome $\mathrm{C}$ (cytC) can be explained by

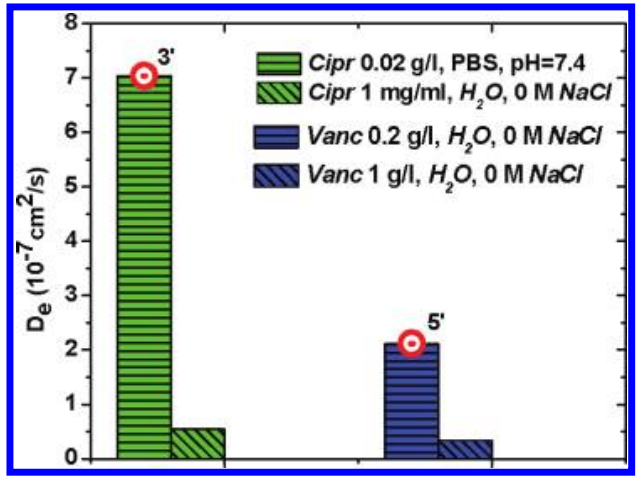

Figure 10. Effective diffusion coefficient for ciprofloxacin and vancomycin through the nanoporous membranes at different conditions. The red open circles represent the $D_{e}$ values used to calculate the constrictivities of the two solutes shown by the same symbols in Figure 9.

the fact that the hydrophobic interaction between proteins and the hydrophobic membrane are significant. At the pH 7.4 of the PBS buffer (see Table 3) cytC is positively charged, the isoelectric point for cytC being 9.8. ${ }^{50}$ The long-range electrostatic interaction is effectively screened by the PBS buffer. In the presence of an uncharged surface like cross-linked 1,2-PB, the adsorption of cytC should stop at the monolayer stage, as reported from many studies. ${ }^{50,51}$ Therefore, a monolayer of cytC is expected to adsorb on the pore walls before the steady state permeation of the protein through the membrane is established. Assuming conserved size of adsorbed protein as in free solution, the effective pore radius would then decrease to $2.1 \mathrm{~nm}$ and the actual $R_{\mathrm{s}} / R_{\mathrm{p}}$ value correspondingly increase to 0.70 . This is the abscissa of the new data point $6^{\prime}$ in Figure 9 , which now follows the prediction of the BB model. Similar considerations on the bigger proteins myoglobin and albumin would spawn the prediction of complete pore clogging and no permeation through the nanopores, which also is in line with our observations.

The influence of physicochemical parameters on the solute transport through the nanoporous membranes was further investigated in the case of ciprofloxacin (cipr) $\left(\mathrm{p} K_{\mathrm{a} 1}=6, \mathrm{p} K_{\mathrm{a} 2}=9\right)$ and vancomycin (vanc; $\mathrm{p} K_{\mathrm{a} 1}=2.2, \mathrm{p} K_{\mathrm{a} 2}=7.8, \mathrm{p} K_{\mathrm{a} 3}=8.9$ ). $\mathrm{A} 13$-fold increase of the effective diffusion coefficient $D_{e}$ for cipr was observed at a $0.02 \mathrm{~g} / \mathrm{L}$ concentration in PBS buffer of $\mathrm{pH}=7.4$. From the $\mathrm{p} K_{\mathrm{a}}$ values given above, cipr is expected to become a zwitterion with zero net charge at such $\mathrm{pH}$, which brings it at similar conditions to the case of ampicillin sodium salt in DI water. Surprisingly, the $D_{e}$ of vanc increased more than 6-fold by a simple 5 -fold dilution in DI water, as can be seen in Figure 10. The increase of ionic strength by addition of $0.15 \mathrm{M} \mathrm{NaCl}$ did not produce noticeable changes (data not shown). However, given the net charge of vanc at $\mathrm{pH} 4.5$, we would have expected a clear effect of charge screening on membrane permeability. A lower concentration creates more free space between solute molecules, 
reducing the effect of electrostatic interaction or hydrogen bonding. The extended planar shape of vanc (see Table S3) would promote molecular stacking stabilized by hydrogen bonds and $\pi-\pi$ interactions (see Table S3, SI), which are effectively reduced by dilution. A common structural feature of the three antibiotic molecules ampicillin, ciprofloxacin and vancomycin at conditions where they all show "BB-like" constrictivity values is that they bear charges close to the molecules' ends: $1^{+}, 1^{-}$at each end of the first two, having a prolongated shape, and $1^{+}, 1^{+}, 1^{-}$at the tips of the roughly triangular-shaped vanc (see Table S3). This charge distribution eliminates any surfactant-like behavior of the molecules, minimizing adsorption at the neutral hydrophobic pore walls. In conclusion, the highest $D_{e}$ values for cipr and vanc, shown by the open circles in Figure 10, were used to calculate constrictivities for the two solutes, as shown by the data points $3^{\prime}$ and $5^{\prime}$ in Figure 9. By altering physicochemical parameters, the diffusion of ciprofloxacin and vancomycin can be changed to follow the BB model!

\section{CONCLUSIONS}

Gyroid nanoporous 1,2-PB membranes prepared from 1,2-PB-b-PDMS block copolymers show high structural uniformity in the bulk, isotropically percolating porosity with no need for prealignment and easy control of pore wall chemistry, therefore, tunable solvent affinity. The outer surface morphology can be controlled through variation of the interface energy by suitable choice of substrates in the process of membrane fabrication. Opened nonskin nanoporous surface was generated using a glass substrate, while a dense skin layer was created in the presence of a fluorinated substrate, or nitrogen gas. Diffusion profiles for the glucose transport through nonskin, single-skin, and double-skin membranes were presented and interpreted by a simple model of defects on the skin layer(s). The model was qualitatively confirmed by flow visualization experiments. It was shown that the permeation rate of water solutions can also be tuned by generating patterned hydrophilized regions of the membranes. The selectivity of the nanoporous membranes was investigated relative to a series of antibiotics, proteins and other biomolecules. The solute transport was discussed in terms of size exclusion and hydrodynamic interactions. The demonstrated flexible diffusion renders this type of nanoporous membranes interesting for various applications. For example, the skin membranes with a low flux might be useful for sensor applications where diffusion restriction is needed. The hydrophilized nonskin membranes might be promising for hemodialysis applications where high flux and antifouling are desired. The study of the performance of the gyroid 1,2-PB membranes as ultrafiltration membranes will be reported separately.

\section{METHODS}

Membrane Preparation. The 1,2-PB-b-PDMS copolymer was synthesized by living anionic polymerization as described in ref 17 . The general procedure to prepare a nanoporous 1,2-PB membrane is as follows. Two different substrates were used for fabricating the flat sheet membrane. One is a glass plate and the other is a FDTS-coated glass plate via molecular vapor deposition. ${ }^{52}$ FDTS stands for $\mathrm{Cl}_{3} \mathrm{Si}\left(\mathrm{CH}_{2}\right)_{2}\left(\mathrm{CF}_{2}\right)_{7} \mathrm{CF}_{3}$. The 1,2-PB-b-PDMS precursor was dissolved in tetrahydrofuran (THF, Sigma-Aldrich) containing 0.01 mols of dicumyl peroxide cross-linker (DCP, Sigma-Aldrich) per mole of 1,2-PB repeating units. For each membrane sheet to be prepared, the solution was cast onto a clean substrate (glass or FDTS-coated glass), followed by drying under nitrogen flow first and then in a vacuum at room temperature. The dried sample was then covered with a second plate (glass or FDTS-coated glass). The two plates were squeezed together under 4 bar in a pneumatic-drive compressing setup under vacuum for $2 \mathrm{~h}$ at room temperature. The thickness of the sample was controlled with a few pieces of $0.5 \mathrm{~cm}$ wide aluminum spacers. The sandwiched block copolymer samples were cross-linked at $140^{\circ} \mathrm{C}$ for $2 \mathrm{~h}$ under nitrogen atmosphere. After cross-linking, the circular flat sheets were removed from the plates and immersed in tetra- $n$-butylammonium fluoride solution (TBAF, Sigma-Aldrich) in THF to selectively and quantitatively remove the PDMS at room temperature. The etched samples were rinsed by a mixture of THF and methanol and further dried under nitrogen flow at room temperature. Membrane disks $1 \mathrm{~cm}$ in diameter were cut out of the flat membrane sheet for the diffusion tests.

Characterization. Atomic force microscopy (AFM) was performed at ambient conditions using NanoMan AFM in tapping mode, with NANOSENSORS SSS-NCH AFM probe. The scan area was $1 \times 1 \mu \mathrm{m}^{2}$ and $512 \times 512$ pixels. The sample to be measured was cut with a blade. The cutting surface was trimmed flat and further microtomed on a Leica ultramicrotome with a cryo $35^{\circ}$ diamond knife (DIATOME) at room temperature. The microtomed sample was glued on a silicon plate for AFM measurement.

Transmission electron microscopy (TEM) was performed on a FEI TECNAI T20 at acceleration voltage of $200 \mathrm{kV}$. The $90 \mathrm{~nm}$ slices of nanoporous 1,2-PB were sectioned on the Leica ultramicrotome with the cryo $35^{\circ}$ diamond knife at room temperature. The slices were deposited onto a holey carbon-coated copper grid for TEM measurements.

Scanning electron microscopy (SEM) was done either on a HELIOS or a Quanta 200F instrument from FEl using an acceleration voltage of 5 or $3 \mathrm{kV}$. The samples were sputter-coated with $2 \mathrm{~nm}$ thick Pt/Pd prior to SEM imaging.

Contact angle measurements (CA) and surface tension measurements were conducted on a Contact 
Angle System OCA 20. Contact angles of water on a glass plate or a FDTS-coated glass plate were measured at room temperature with the sessile drop method. ${ }^{53}$

X-ray photoelectron spectroscopy (XPS) was recorded on a Surface Science Instruments Sage-100 with a monochromated Al K $\alpha$ X-ray at takeoff angle of $90^{\circ}$. Elements present on the surface were identified from a survey spectrum over the energy range $0-1400$ $\mathrm{eV}$ with pass energy of $100 \mathrm{eV}$ and resolution of $0.5 \mathrm{eV}$. The spectrum was analyzed with the software Avantage provided by the manufacturer.

Diffusion Tests. The diffusion cells contain two compartments separated by the membrane disk to be tested. The tested membrane was placed onto an O-ring and sealed into the two chambers. Once the membrane was in place, we first checked the liquid leakage by filling deionized water in the feed chamber for $1 \mathrm{~h}$. No liquid leakage was observed. Prior to the diffusion tests, both chambers were loaded with ethanol to prewet the hydrophobic membrane for $30 \mathrm{~min}$. The deionized water was then filled to replace ethanol for another $30 \mathrm{~min}$. After prewetting, the feed chamber was set with $5 \mathrm{~mL}$ solution to be tested; the permeate chamber was loaded with the same volume of pure solvent. Both chambers were stirred by standard Teflon magnetic stirrers throughout the entire experiment. Equal amount of solutions were withdrawn from both chambers at planned time intervals. The solutes' concentration (hydrogen peroxide and glucose) in the receiver cell was determined using a commercial electrochemical analyzer at Radiometer ApS; the concentration of the other solutes was determined using an Ultrospec 3000 UV/visible spectrophotometer (Pharmacia Biotech Ltd., Cambridge, U.K.). All the tested solutes but cypr $\cdot \mathrm{HCl} \cdot \mathrm{H}_{2} \mathrm{O}$ (from Bayer Pharmaceutical) are purchased from Sigma-Aldrich and used as received. They are listed in Table 3 together with specific experimental conditions. See Table S3 for chemical formulas and a number of $\mathrm{p} K_{\mathrm{a}}$ and solubility values.

Acknowledgment. The authors thank L. Hubert at DTUNanotech for the XPS measurements, T. W. Hansen at DTUCEN for the TEM images, A. B. da Silva Fanta at DTU-CEN for the SEM image of Figure 2b, and N. Rozlosnik at DTU-Nanotech for help with the optical microscope. L. Li acknowledges Radiometer Medical ApS and the Danish Council for Technology and Innovation for cofunding her Ph.D. scholarship.

Supporting Information Available: SAXS image of the gyroid bulk porosity; AFM images of bulk section and of nonskin upper surface; TEM of grain boundaries in the bulk; SEM of skin membrane surfaces; XPS analysis; Model of nanoporous membrane with cylindrical morphology; Flow visualization; Bungay and Brenner equation; Chemical structure of solutes used in the selectivity study. This material is available free of charge via the Internet at http://pubs.acs.org.

\section{REFERENCES AND NOTES}

1. Stamatialis, D. F.; Papenburg, B. J.; Girones, M.; Saiful, S; Bettahalli, S. N. M.; Schmitmeier, S.; Wessling, M. Medical Applications of Membranes: Drug Delivery, Artificial Organs and Tissue Engineering. J. Membr. Sci. 2008, 3, 1-34.
2. Bazinet, L.; Lamarchey, F.; Ippersiel, D. Bipolar-Membrane Electrodialysis: Applications of Electrodialysis in the Food Industry. Trends Food Sci. Technol. 1998, 9, 107-113.

3. Visvanathan, C.; Aim, R. B.; Parameshwaran, K. Membrane Separation Bioreactors for Wastewater Treatment. Crit. Rev. Environ. Sci. Technol. 2000, 30, 1-48.

4. Peinemann, K. V.; Nunes, S. P. Membrane Technology, Vol. 1: Membranes for Energy Conversion; Wiley-VCH Verlag $\mathrm{GmbH} \&$ Co. KGaA: Weinheim, 2008.

5. Ulbricht, M. Advanced Functional Polymer Membranes. Polymer 2006, 47, 2217-2262.

6. Ho, A. K.; Perera, J. M.; Dunstan, D. E.; Stevens, G. W. Measurement and Theoretical Modeling of Protein Mobility through Membranes. AIChE J. 1999, 45, 1434-1450.

7. Thormann, A.; Teuscher, N.; Pfannmöller, M.; Rothe, U.; Heilmann, A. Nanoporous Aluminum Oxide Membranes for Filtration and Biofunctionalization. Small 2007, 3, 1032-1040.

8. Bates, F. S. Block Copolymer Thermodynamics: Theory and Experiment. Annu. Rev. Phys. Chem. 1990, 41, 525-557.

9. Albrecht, T. T.; Steiner, R.; DeRouchey, J.; Stafford, C. M.; Huang, E; Bal, M.; Tuominen, M.; Hawker, C. J.; Russell, T. P. Nanoscopic Templates from Oriented Block Copolymer Films. Adv. Mater. 2000, 12, 787-791.

10. Ting, Y. H.; Park, S. M.; Liu, C. C.; Liu, X. S.; Himpsel, F. J.; Nealey, P. F.; Wendt, A. E. Plasma Etch Removal of Poly(methyl methacrylate) in Block Copolymer Lithography. J. Vac. Sci. Technol., Part B 2008, 26, 1071-1023.

11. Hashimoto, T; Tsutsumi, K; Funaki, Y. Nanoprocessing Based on Bicontinuous Microdomains of Block Copolymers: Nanochannels Coated with Metals. Langmuir 1997, 13, 6869-6872.

12. Zalusky, A. S.; Valles, O. R.; Wolf, J. H.; Hillmyer, M. A. Ordered Nanoporous Polymers from Polystyrene-Polylactide Block Copolymers. J. Am. Chem. Soc. 2002, 124, 12761-12773.

13. Xu, T.; Stevens, J.; Villa, J. A.; Goldbach, J. T.; Guarini, K. W.; Black, C. T.; Hawker, C. J.; Russell, T. P. Block Copolymer Surface Reconstuction: A Reversible Route to Nanoporous Films. Adv. Funct. Mater. 2003, 13, 698-702.

14. Guo, F.; Jankova, K.; Schulte, L.; Vigild, M. E.; Ndoni, S. OneStep Routes from Di- and Triblock Copolymer Precursors to Hydrophilic Nanoporous Poly(acrylic acid)-b-Polystyrene. Macromolecules 2008, 41, 1486-1493.

15. Ndoni, S.; Vigild, M. E.; Berg, R. H. Nanoporous Materials with Spherical and Gyroid Cavities Created by Quantitative Etching of Polydimethylsiloxane in PolystyrenePolydimethylsiloxane Block Copolymers. J. Am. Chem. Soc. 2003, 125, 13366-13367.

16. Hansen, M. S.; Vigild, M. E.; Berg, R. H.; Ndoni, S. Nanoporous Crosslinked Polyisoprene from a PolyisoprenePolydimethylsiloxane Block Copolymer. Polym. Bull. 2004, 51, 403-409.

17. Schulte, L.; Grydgaard, A.; Jakobsen, M. R.; Szewczykowski, P. P.; Guo, F.; Vigild, M. E.; Berg, R. H.; Ndoni, S. Nanoporous Materials from Stable and Metastable Structures of 1,2-PBb-PDMS Block Copolymers. Polymer 2011, 52, 422.

18. Orilall, M. C.; Wiesner, U. Block Copolymer Based Composition and Morphology Control in Nanostructured Hybrid Materials for Energy Conversion and Storage: Solar Cells, Batteries and Fuel Cells. Chem. Soc. Rev. 2011, 40, 520-535.

19. Guo, F.; Jankova, K.; Schulte, L.; Vigild, M. E.; Ndoni, S. Surface Modification of Nanoporous 1,2-Polybutadiene by Atom Transfer Radical Polymerization or Click Chemistry. Langmuir 2010, 26, 2008-2013.

20. Ndoni, S.; Li, L.; Schulte, L.; Szewczykowski, P. P.; Hansen, T. W.; Guo, F. X.; Berg, R. H.; Vigild, M. E. Controlled Photooxidation of Nanoporous Polymers. Macromolecules 2009, 42, 3877-3880.

21. Berthold, A; Sagar, K.; Ndoni, S. Patterned Hydrophilization of Nanoporous 1,2-PB by Thiol-Ene Photochemistry. Macromol. Rapid Commun. 2011, 32, 1259-1263.

22. Hillmyer, M. A. Nanoporus Materials from Block Copolymer Precursors. Adv. Polym. Sci. 2005, 190, 137-181.

23. Jackson, E. A.; Hillmyer, M. A. Nanoporous Membranes Derived from Block Copolymers: From Drug Delivery to Water Filtration. ACS Nano 2010, 4, 3548-3553. 
24. Yang, S. Y.; Ryu, I.; Kim, H. Y.; Kim, J. K.; Jang, S. K.; Russell, T. P. Nanoporous Membranes with Ultrahigh Selectivity and Flux for the Filtration of Viruses. Adv. Mater. 2006, 18, 709-712.

25. Yang, S. Y.; Yang, J. A.; Kim, E. S.; Jeon, G.; Oh, E. J.; Choi, K. Y.; Hahn, S. K.; Kim, J. K. Single-File Diffusion of Protein Drugs Through Cylindrical Nanochannels. ACS Nano 2010, 4, 3817-3822.

26. Lo, K. H.; Chen, M. C.; Ho, R. M.; Sung, H. W. Pore-Filling Nanoporous Templates from Degradable Block Copolymers for Nanoscale Drug Delivery. ACS Nano 2009, 3, 2660-2666.

27. Phillip, W. A.; O'Neill, B.; Rodwogin, M.; Hillmyer, M. A.; Cussler, E. L. Self-Assembled Block Copolymer Thin Films As Water Filtration Membranes. Appl. Mater. Interfaces 2010, 2, 847-853.

28. Uehara, H.; Kakiage, M.; Sekiya, M.; Sakuma, D.; Yamonobe, T.; Takano, N.; Barraud, A.; Meurville, E.; Ryser, P. SizeSelective Diffusion in Nanoporous but Flexible Membranes for Glucose Sensors. ACS Nano 2009, 3, 924-932.

29. Mansky, P.; Russell, T. P.; Hawker, C. J.; Pitsikalis, M.; Mays, J. Ordered Diblock Copolymer Films on Random Copolymer Brushes. Macromolecules 1997, 30, 6810-6813.

30. Bodas, D.; Malek, C. K. Formation of More Stable Hydrophilic Surfaces of PDMS by Plasma and Chemical Treatments. Microelectron. Eng. 2006, 83, 1277-1279.

31. Cassie, A. B. D.; Baxter, S. Wettability of Porous Surfaces. Faraday Soc. 1944, 40, 546-551.

32. Keister, J. C.; Berner, B. Permeation Through Membranes with a Discontinuous Impermeable Surface Coating. J. Controlled Release 1986, 3, 155-166.

33. Epstein, N. On Tortuosity and the Tortuosity Factor in Flow and Diffusion Through Porous Media. Chem. Eng. Sci. 1989, 44, 777-779.

34. Langsdorf, L. J.; Zydney, A. L. Diffusive and Convective Solute Transport Through Hemodialysis Membranes: A Hydrodynamic Analysis. J. Biomed. Mater. Res. 1994, 28, 573-582.

35. Henzler, T.; Steudle, E. Transport and Metabolic Degradation of Hydrogen Peroxide in Chara Corallina: Model Calculations and Measurements with the Pressure Probe Suggest Transport of $\mathrm{H}_{2} \mathrm{O}_{2}$ Across Water Channels. J. Exp. Bot. 2000, 51, 2053-2066.

36. Stewart, P. S. Diffusion in Biofilms. J. Bacteriol. 2003, 185, 1485-1491.

37. Suci, P. A.; Mittelman., M. W.; Yu, F. P.; Geesey, G. G. Investigation of Ciprofloxacin Penetration into Pseudomonas Aeruginosa Biofilms. Antimicrob. Agents Chemother. 1994, 38, 2125-2133.

38. Arabski, M.; Wasik, S.; Dworecki, K.; Kaca, W. Laser Interferometric Determination of Ampicillin and Colistin Transfer Through Cellulose Biomembrane in the Presence of Proteus Vulgaris $\mathrm{O} 25$ Lipopolysaccharide. J. Membr. Sci. 2007, 299, 268-275.

39. Bleicher, K.; Lin, M.; Shapiro, M. J.; Wareing, J. R. Diffusion Edited NMR: Screening Compound Mixtures by Affinity NMR to Detect Binding Ligands to Vancomycin. J. Org. Chem. 1998, 63, 8486-8490.

40. Gupte, S. S.; Charles, R. H. Multidimensional Diffusion Modes and Collision Frequencies of Cytochrome $C$ with Its Redox Partners. J. Biol. Chem. 1988, 263, 5241-5247.

41. Moreno, V. R.; Wittenberg, J. B. The Self-Diffusion Coefficients of Myoglobin and Hemoglobin in Concentrated Solutions. J. Biol. Chem. 1972, 247, 895-901.

42. Fardet, A.; Hoebler, C.; Djelveh, G.; Barry, J. L. Restricted Bovine Serum Albumin Diffusion Through the Protein Network of Pasta. J. Agric. Food Chem. 1998, 46, 4635-4641.

43. Dechadilok, P.; Deen, W. M. Hindrance Factors for Diffusion and Convection in Pores. Ind. Eng. Chem. Res. 2006, 45, 6953-6959.

44. Milhaupt, J. M.; Lodge, T. P. Homopolymer and SmallMolecule Tracer Diffusion in a Gyroid Matrix. J. Polym. Sci., Part B: Polym. Phys. 2001, 39, 843-859.

45. Li, L.; Szewczykowski, P.; Clausen, L. D.; Hansen, K. M.; Jonsson, G. E.; Ndoni, S. Ultrafiltration by Gyroid Nanoporous Polymer Membranes. J. Membr. Sci. 2011, doi: 10.1016/j.memsci.2011.09.012.
46. Bungay, P. M.; Brenner, H. The Motion of a Closely Fitting Sphere in a Fluid-Filled Tube. Int. J. Multiphase Flow 1973, 1, 25-56.

47. Ghosh, R.; Cui, Z. F. Fractionation of BSA and Lysozyme Using Ultrafitration: Effect of $\mathrm{pH}$ and Membrane Pretreatment. J. Membr. Sci. 1998, 139, 17-28.

48. Deen, W. M. Hindered Transport of Large Molecules in Liquid-Filled Pores. AIChE J. 1987, 33, 1409-1425.

49. Latulippea, D. R.; Mikab, A. M.; Childsb, R. F.; Ghosha, R.; Filipe, C. D. M. Flux Performance and Macrosolute Sieving Behavior of Environment Responsive Formed-in-Place Ultrafiltration Membranes. J. Membr. Sci. 2009, 342, 227-235.

50. Vinu, A.; Murugesan, V.; Tangermann, O; Hartmann, M. Adsorption of Cytochrome $C$ on Mesoporous Molecular Sieves: Influence of $\mathrm{pH}$, Pore Diameter, and Aluminium Incorporation. Chem. Mater. 2004, 16, 3056-3065.

51. Valetta, P.; Thomas, M.; Déjardin, P. Adsorption of Low Molecular Weight Proteins to Hemodialysis Membranes: Experimental Results and Simulations. Biomaterials 1999, 20, 1621-1634.

52. Murillo, G.; Davis, Z. J.; Keller, S.; Abadal, G.; Agusti, J.; Cagliani, A.; Noeth, N.; Boisen, A.; Barniol, N. Novel SU-8 Based Vacuum Wafer-Level Packaging for MEMS Devices. Microelectron. Eng. 2010, 87, 1173-1176.

53. Gaines, G. L. Surface and Interfacial Tension of Polymer Liquids. Polym. Eng. Sci. 1972, 12, 1-11. 